\title{
A Unified Account of Three Uses of Dou*
}

\author{
Zhuo Chen \\ The Graduate Center \\ The City University of New York
}

\begin{abstract}
I argue that dou is a distributor that always distributes through events, based on the following three pieces of evidence: (a) dou has an occasion reading; (b) the occasion reading is obligatory when the NP outside the scope of dou is semantically singular; (c) two NP pluralities outside of the scope of dou generate a cumulative reading. Among these three, the first one is often ignored in the literature. The second one directly debunks the mystery of the incompatibility between $d o u$ and collective predicates and the third one poses a challenge to the current popular theories on dou on the market. Following Champollion's (2016) analysis of overt distributors cross-linguistically, I analyze dou as a distributor over events. What's inside the scope of dou is the description of the subevents and what's outside its scope is the description of the mereological sum event. This analysis can give a unified account of the distributive use, occasion use and cumulative use of $d o u$.
\end{abstract}

Keywords: $D o u$, distributivity, cumulativity, event semantics

\section{Introduction}

\subsection{Background}

The Chinese particle dou has attracted immense interest due to its multi-function. The classical analysis treats $d o u$ as an overt counterpart of the covert dist proposed in Schwarzschild 1996 (Lin 1998). Subsequent research analyzes dou as a maximality operator which basically functions as English the (Xiang 2008; Cheng \& Giannakidou 2013). More recent research takes the even use of dou as the starting point and extends the analysis to the distributive use by releasing the task of distribution to an invisible element in the sentence (Liao 2011; Liu 2017).

* I thank Sam Alxatib for comments and discussion on this project. I also thank Danny Fox for his insightful comments on the examples of the cumulative use of dou. Thanks to Irene Heim for pointing out a mistake in my composition and to Lucas Champollion for proposing a possible solution. Thanks to the audience at SALT28 for their comments and questions. A special thanks to Roger Schwarzschild whose lectures on event semantics inspired this project. All errors and inadequacies in this paper are mine.

(O)2018 Chen 
Another line of research that does not draw much attention relates dou to event quantification. Two representative proposals are Huang (1996) and Li (1995). Huang (1996) argues that a set of quantifiers including every and most require an existential quantifier in their scope to facilitate distribution. Assuming that predicates introduce a variable (Higginbotham 1985, 1989), she argues dou serves as an existential closure (Heim 1982) to existentially bind the event variable. Li (1995) also proposes that dou is an event quantifier, but he preserves the universal quantificational force of dou and locates the event variable to the left of it in order to maintain the Leftness Condition on $\mathrm{dou}^{1}$

\subsection{Data and questions}

The data that will be discussed in the current paper are confined to the following. Even reading of dou is set aside for now.

(1) Wo dou du le Laozhongshi

I DOU bet Asp Old Faithful

I bet on Old Faithful every time. Occasion reading only ${ }^{2}$

(2) Yuehan he Mali dou jianmian le

John and Mary DOU meet Asp

John and Mary met each other each time/at each place.

Occasion reading only ${ }^{3}$

(3) Tamen dou taiqi le wu jia gangqin

they DOU lift Asp five CL piano

They each lifted five pianos.

$\checkmark$ Distributive reading

They lifted five pianos each time/at each place.

$\checkmark$ Occasion reading

(4) Yuehan he Mali dou xihuan Bier

John and Mary DOU like Bill

John and Bill both like Bill.

Distributive reading only

*?Yuehan dou xihuan Bier

John DOU like Bill

John likes Bill every time/at each place. $\quad$ *Occasion reading 4

1 The Leftness Condition (Cheng 1994): dou always quantifies over the element to its left.

2 This datum is from Li (1995). In a context where the speaker is talking about the multiple horse races he bet on last week, this sentence means he bet on Old Faithful in all those races.

3 'John and Mary' is semantically singular, in the sense of Landman (2000), where a morphologically plural NP with a collective predicate will be turned into a semantic impure atom by the $\uparrow$ operator.

4 Note that the even reading of $d o u$ is felicitous here, but as I said, this use is set aside for now. 
*?Yuehan/Yuehan he Mali dou dapo le na ge beizi

John/John and Mary DOU break Asp that CL cup

John broke that cup every time/at every place.

John and Mary each broke that cup.
*Occasion reading

*Distributive reading

(7) San ge xuesheng ba wu ge pingguo dou chi le three CL students BA five CL apple DOU eat Asp

Three students ate all of the five apples.

${ }^{O K}$ Distributive reading $\checkmark$ Cumulative reading

Observation of these data leads us to ask the following questions:

- When the NP outside of the scope of dou is semantically singular, why is the occasion reading obligatory, for example, in (1) and (2)?

- When the NP outside of the scope of dou is semantically plural and the VP denotes pluralizable events, why are distributive reading and occasion reading both possible, for example, in (3)?

- When the NP outside of the scope of dou is semantically plural and the VP is an individual-level predicate, why is distributive reading the only possible reading, for example, in (4)?

- When the NP outside of the scope of dou is semantically singular and the VP is an individual-level predicate, why is the occasion reading prohibited, for example, in (5)?

- When the VP denotes non-pluralizable events, why is the sentence bad on both the occasion reading and the distributive reading, for example, in (6)?

- When both the subject NP and object NP are outside of the scope of dou and both are semantically plural, how do we get the cumulative reading, for example, in (7)?

Several parameters emerge from the data whose interaction determines the grammaticality and possible readings of the examples, including the semantic singularity/plurality of the NP, the stage/individual level of the predicate and if the predicate is stage-level, whether it denotes pluralizable events. Putting aside the cumulative reading, the interaction between the different settings and the resulting reading they lead to are shown in Table 1. 


\begin{tabular}{|c|c|c|c|}
\hline $\begin{array}{c}\text { NP } \\
\text { semantically } \\
\text { plural }\end{array}$ & $\begin{array}{c}\text { Stage-level } \\
\text { pluralizable } \\
\text { predicates }\end{array}$ & $\begin{array}{c}\text { Stage-level } \\
\text { non-pluralizable } \\
\text { predicates }\end{array}$ & Reading \\
\hline+ & + & - & distributive, occasion \\
\hline+ & - & + & ungrammatical \\
\hline+ & - & - & distributive only \\
\hline- & + & - & occasion only \\
\hline$-/+$ & - & + & ungrammatical \\
\hline- & - & - & ungrammatical \\
\hline
\end{tabular}

Table 1 Data

As for the cumulative reading, we need to answer: when the object is in the scope of $d o u$, why do we only get a distributive reading on the subject NP or an occasion reading? When the object is outside the scope of $d o u$, how does the cumulative reading become available? Compare (8) to (3).

Tamen ba wu jia gangqin dou taiqi le they BA five CL piano DOU lift Asp

They lifted all the five pianos.

$\checkmark$ Cumulative reading

\subsection{The scope and organization of this paper}

The modest goal of this paper is to revive the idea of $d o u$ as an event quantifier (Huang 1996; Li 1995). In section 2, I will show that the cumulative use poses a challenge to Lin's (1998) and Liu's (2017) analyses. Two attempts to defend their analyses will be discussed and problems with these attempts are pointed out. In section 3, I propose that dou is a distributor that always distributes through events. A semantic entry will be given to dou that adds it to the category of distance distributive items surveyed in Champollion 2016. This semantic analysis is shown to be able to unify the distributive use, occasion use and cumulative use of dou. The questions that arise from the data in section 1 will also find their answers in this analysis. Section 4 concludes.

\section{Cumulative use of $d o u$}

Despite the huge literature on $d o u$, the cumulative use of it has been largely ignored. Sentence (7), repeated here as (9), is an example. In the given scenario where the three students stand in a cumulative relationship with the five apples, (9) is true. 
(9) San ge xuesheng ba wu ge pingguo dou chi le three CL students BA five CL apples DOU eat Asp Three students ate all the five apples.

(10) Scenario: John, Mary and Bill are three students. On the table there were five apples. John ate two, Bill ate two and Mary ate one.

If the previous semantic analyses of $d o u$ can be successfully extended to account for the cumulative use of $d o u$, examples like (9) are positive evidence to support them. If they cannot explain the cumulative use, we need to make the semantics of dou more flexible as to accommodate examples like (9). In the following two sections, I will show that the cumulative reading poses a challenge to Lin's (1998) overt dist analysis and Liu's (2017) even analysis.

\subsection{Challenge to Dou as an overt dist}

Lin (1998), following Schwarzschild's (1996) analysis of the covert distributive operator dist in English, proposes that dou is simply an overt version of it. In this proposal, the semantics of dou involves three parameters, a predicate, an NP to be distributed over, and a contextually determined cover on the NP. Dou asserts that the predicate applies to each element in the cover. For exposition, the definition of cover and the semantics of dou are given in (11) and (12). The contextually determined cover is returned by applying to a contextually given function Cov to the NP. With these parameters available, we can account for a distributive sentence straightforwardly. (13) is an example.

(11) a. $\mathrm{C}$ is a plurality cover of $\mathrm{A}$ iff $\mathrm{C}$ covers $\mathrm{A}$ and no proper subset of $\mathrm{C}$ covers A

b. $\mathrm{C}$ covers $\mathrm{A}$ if:

1) $C$ is a set of subsets of $A$

2) Every member of A belongs to some set in $C$

3) $\emptyset$ is not in $\mathrm{C}$

$$
\llbracket d o u \rrbracket=\lambda \operatorname{Cov} \lambda P \lambda x . \forall y(y \in \operatorname{Cov}(x) \rightarrow P(y))
$$

【The students DOU bought a car》 $=1$ iff $\forall y(y \in \operatorname{Cov}(\llbracket$ the students $\rrbracket) \rightarrow \llbracket$ bought a $\operatorname{car} \rrbracket(y)=1)$

Can this analysis capture the cumulative sentence in (9)? According to Lin's (1998) proposal, dou distributes over a cover on the NP five apples in this sentence. Suppose the contextually cover is as in (14). The resulting meaning is as shown in $(15)^{5}$, which asserts that there are three students, and for each of the five apples, the

5 We take BA as the thematic role head that introduces the preverbal object. What its syntactic category 
three students ate it. This is a felicitous meaning, but not the cumulative reading that we are targeting. In our reading, it is not the case that all the three students are involved in eating each of the five apples. ${ }^{6}$

$\operatorname{Cov}(\llbracket$ the five apples $\rrbracket)=\left\{\left\{a_{1}\right\},\left\{a_{2}\right\},\left\{a_{3}\right\},\left\{a_{4}\right\},\left\{a_{5}\right\}\right\}$

(15) «Three students five apples DOU ate $=1$ iff $\exists X(\llbracket$ students $\rrbracket(X) \wedge|X|=3 \wedge$

$\forall y(y \in \operatorname{Cov}(\llbracket$ the five apples $\rrbracket) \rightarrow \llbracket$ ate $\rrbracket(y)(X)=1))$

\subsection{Challenge to Dou as even}

Liu (2017) approaches the semantics of dou from a different direction than Lin (1998). To Liu, dou is never a distributor by itself. Instead, the semantic core of dou is analogous to even. The distributive meaning is contributed by a covert dist as proposed in Schwarzschild 1996. I summarize the main components of Liu's (2017) theory as follows.

The main components in Liu's (2017) theory

- Dou is a focus particle with a presuppositional semantics similar to English even $\llbracket \mathrm{Dou} \rrbracket=\lambda p . \forall q\left(q \in \operatorname{alt}(p) \rightarrow p<_{\text {likely }} q\right): p$

- A covert dist is present in distributive sentences containing dou.

- In distributive sentences, dou activates atom-based alternatives to its focus associate while in even sentences, dou activates group-based alternatives. A consequence of the different types of alternatives is that the even flavor gets trivialized in distributive sentences.

Let's understand how this theory works using (17) and (19) for illustration. In (17), suppose there are three students in the context, $s_{1}, s_{2}$ and $s_{3}$. The prejacent of dou is logically strongest in the alternative set and entails all the other alternatives. This logical entailment relationship between the alternatives can be represented as a lattice, shown in (18).

is and whether it forms a constituent with the NP following it has been debated. For a review on the literature on BA, see Huang, Li \& Li 2009. In this paper, what matters for us is the fact that the BA-phrase preceding dou confines association of dou to the NP within it. The discussion on this fact can be found in Lee (1986).

6 Note that a singleton cover $\left\{\left\{a_{1}, a_{2}, a_{3}, a_{4}, a_{5}\right\}\right\}$ is compatible with the cumulative reading, yet it's prohibited. This prohibition is exhibited in the impossible interpretation of 'John and Mary DOU got married' as 'John and Mary married each other'. One possible argument for this prohibition is that Gricean maxim Be Brief rules out the singleton cover because the sentence meaning stays the same with or without dou when a singleton cover is adopted. 
(17) The students DOU bought a car.

The students each bought a car.

LF: [DOU [the students dist bought a car]]

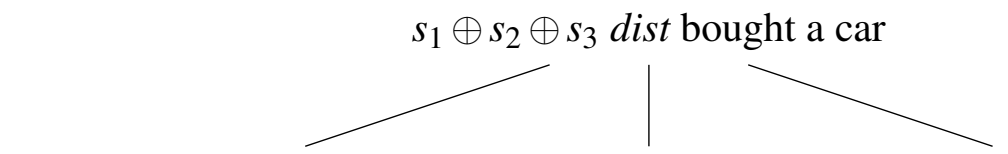

$s_{1} \oplus s_{2}$ dist bought a car $\quad s_{1} \oplus s_{3}$ dist bought a car $\quad s_{2} \oplus s_{3}$ dist bought a car



To Liu, logical entailment is stronger than likelihood. If $p$ logically entails $q, p$ is at least as unlikely as $q$ regardless of the context. Given the fact that the prejacent of dou in (17) entails the other alternatives, Liu (2017) argues that the presupposition contributed by dou is always automatically satisfied in distributive sentences and this is why we cannot sense the even flavor.

In even sentences like (19), Liu (2017) proposes that dist is absent and alternatives are turned into group-based using the $\uparrow$ operator suggested in Landman (2000). A result from these two changes is that the prejacent of $d o u$ is logically independent from the other alternatives. In order to satisfy the presupposition of $d o u$, the context has to be one in which the prejacent is least likely among the alternatives. This is the reason why we can sense the even flavor.

The students DOU bought a car.

Even the students bought a car.

LF: [DOU [the students bought a car]]

$$
\uparrow s_{1} \oplus s_{2} \oplus s_{3} \text { dist bought a car }
$$

$\uparrow s_{1} \oplus s_{2}$ dist bought a car $\uparrow s_{1} \oplus s_{3}$ dist bought a car $\uparrow s_{2} \oplus s_{3}$ dist bought a car

$$
\uparrow s_{1} \text { bought a car } \quad \uparrow s_{2} \text { bought a car } \quad \uparrow s_{3} \text { bought a car }
$$

Liu's (2017) theory has great merits in unifying the distributive use and even use of dou. However, it also faces a challenge when we turn to the cumulative use of dou. It turns out that neither choice provided in his theory delivers the correct interpretation of the cumulative reading. 
Based on Liu 2017, we have two choices when interpreting (9). First, if dist is present, we will get the same meaning as given in (15). As discussed before, this is a felicitous reading in a context where there are three students who participate in eating each of the five apples, but it's not the cumulative interpretation that we attempt to capture.

(21) Three students BA five apples DOU ate

Three students ate each of the five apples

LF: [DOU [three students five apples dist ate]]

If dist is not present, Liu's theory predicts that we get an even flavor of this sentence. The interpretation of (22) is roughly 'there are three students who ate even the five apples'. A cumulative reading of the prejacent of dou is indeed available. However, due to the fact that group-based alternatives to 'the five apples' generate a set of propositions that do not entail each other, Liu predicts that the even flavor is obligatory. This is not true. The cumulative interpretation of sentences like (22) never requires to be accompanied by an even flavor.

(22) Three students BA five apples DOU ate

Three students ate even the five apples

LF: [DOU [three students five apples ate]]

In the next section, I will present evidence against two arguments that defend the existing theories by reducing the cumulative reading of dou to either non-maximality reading or team-credit reading.

\subsection{Cumulative reading of $\mathrm{dou}$ is not a non-maximality reading}

Non-maximality refers to the observation that predications with definite plurals allow exceptions (Link 2002; Dowty 1987; Brisson 1998). For example, sentence (23) is true in a context where few professors countenance a neutral expression.

(23) The professors smiled.

A reviewer points out that both Lin's (1998) and Liu's (2017) theory can account for the cumulative reading of $d o u$ if we take into consideration the non-maximality reading of definite plurals. For example, the subject the students in (24) stands in a cumulative relationship with the five apples in the given scenario. Lin's (1998) theory on dou as dist will derive a meaning that for each of the five apples, the students ate it. Liu's (2017) theory with the assumption that a covert dist is present will derive the same meaning.

(24) xueshengmen ba wu ge pingguo dou chi le the students BA five CL apples DOU eat Asp 
The students ate all the five apples.

(25) Scenario: there are three students, $s_{1}, s_{2}, s_{3}$ and five apples, $a_{1}, a_{2}, a_{3}, a_{4}, a_{5}$. $s_{1}$ ate $a_{1}$ and $a_{2}, s_{2}$ ate $a_{3}$ and $s_{3}$ ate $a_{4}$ and $a_{5}$.

Literally, the derived meaning is not the cumulative reading we want to obtain. However, given the non-maximality reading of definite plurals, it is allowed to say 'the students ate an apple' when only one of them actually ate it. In the given scenario, even though $s_{1}$ individually ate $a_{1}$ and $a_{2}$, under non-maximality, it is still true to assert that the students ate $a_{1}$ and $a_{2}$. The same reasoning applies to $s_{2}$ and $s_{3}$. Therefore, the original semantic meaning we obtain for this sentence - for each of the five apples, the students ate it — is regarded as true in the given scenario once the subject is read non-maximally.

However, the resort to non-maximality to accommodate the cumulative reading immediately encounters a problem when we replace the subject with an indefinite numeral-classifier phrase or a definite numeral-classifier phrase. Neither allows a non-maximality reading yet both are grammatical in a cumulative sentence with $\mathrm{dou}$. We have seen that (9) is true in a cumulative scenario, but non-maximality does not apply to the subject three students in (9). In contrast to the observation in (23) on definite plurals, we cannot truthfully say that three students ate all the five apples when only two did the eating. The attempt to simply relegate the cumulative reading to the non-maximality reading of definite plurals, therefore, is not general enough to capture (9).

\subsection{Cumulative reading of $d o u$ is not a team-credit reading}

Another defense also questions the status of cumulative reading of $d o u$ as a real reading and tries to reduce it to the team-credit reading. Team-credit reading refers to a reading where an achievement of some of the team members is considered as an achievement of the whole team. For example, in a scenario where some boys participated in building a raft with one or two boys idling away, sentence (26) is still regarded as true.

(26) The boys built a raft.

One advantage of this defense over the non-maximality defense is that teamcredit reading is not confined to definite plurals only. Indefinite numeral phrases also allow it. Example (27) from Kratzer 2002 is true when the three copy editors cumulatively caught all the mistakes. Neither scope relationship between the existential subject and universal object can capture this meaning. However, if any mistake caught by one of the editors is counted as being caught by them three, the cumulative reading is naturally accounted for. 
(27) Three copy editors caught every mistake in the manuscript.

Team-credit reading has its own problems, though. First, the cumulative relationship between the subject and the object in (9) and (24) can be maintained in a context where no team whatsoever is formed between the people denoted by the subject NP. For example in (9), if the three students didn't know each other and they grabbed apples on a table and ate them, the sentence is still true as long as the total number of apples eaten by them adds up to five.

Some people may say that whether the three students have formed a team is determined by the speaker's perception, not the actual matter of fact. This is not unreasonable but can be easily dismissed. In a context where three participants in Jeopardy compete to answer questions, sentence (28) is true when they cumulatively complete all the answers. Despite the fact that competitors don't work as a collaborative team, (28) is still true in a cumulative scenario. Therefore, we can conclude that the cumulative reading of $d o u$ is not simply a team-credit reading.

San ge cansaizhe ba timu dou dawan le three CL participants BA questions DOU answer-finish Asp Three participants answered all the questions.

\subsection{Interim summary}

Given the fact that we can make cumulative examples of dou when neither non-maximality or team-credit reading is satisfied, I conclude that the cumulative reading of dou is an independent reading that cannot be reduced to either of them. The cumulative use of dou being admitted, the challenge posed by it to the distributor analysis in Lin 1998 and the even analysis in Liu 2017 calls for a solution. In the next section, I will propose a semantics of dou that can account for the cumulative reading. Moreover, the new semantics of dou can also capture the distributive use and occasion use of dou. The questions that we ask in $\S 1$ all find an answer in this new analysis of $d o u$.

\section{Dou as an event distributor}

The idea that $d o u$ is an event quantifier has been harbored by a few scholars. In order to resolve the problem of double quantification in sentences containing both a universal quantifier and dou, Huang (1996) proposes that dou is an existential quantifier over the event variable introduced by the predicate.

Li (1995) shares his observation that dou can quantify over the event domain and a sentence with singular NPs and collective predicates can get an occasion reading. To him, dou is a universal quantifier over events and the distribution that 
comes with $d o u$ is to establish a mapping relationship between the subparts of the plural NP and the subevents that dou quantifies over.

The current paper inherits the main idea that dou is an event quantifier but intends to give a compositional semantics to it. Adopting Champollion's (2016) analysis of distributive distance items, I will show how this analysis can account for the distributive, occasion, and cumulative use of dou. Moreover, I will show how this analysis answers the questions we ask in $\S 1$.

\subsection{Compositional semantics of $d o u$}

The analysis presented in this section draws heavily from Champollion's (2016) analysis of overt distributors cross-linguistically. I summarize the components to be included in the semantics of $d o u$ as follows. First, dou can be associated with a plural element that is either overt or covert in the sentence. This association is contextually determined. The achievement of this association is by indexing dou with a thematic function $\theta$. Second, a contextually determined cover on $d o u$ 's associate will be given to $d o u$ as input. When $\theta$ is contextually resolved to a thematic role function, dou distributes over the individuals having that thematic role in the cover. When $\theta$ is contextually resolved to a temporal or spatial trace function, dou distributes over the temporal intervals or spatial locations in the cover. How fine-grained the distribution is down to is also contextually given. Third and the most important component is that $d o u$ is a universal quantifier that always distributes through events. What follows dou in the sentence is the description of the subevents and what precedes it the description of the mereological sum event.

The semantic entry of dou is given in (29). We assume thematic cumulativity as in (30). If John is the agent of $e_{1}$ and Bill is the agent of $e_{2}$, then John $\oplus$ Bill is the agent of $e_{1} \oplus e_{2}$. Following neo-Davidsonian event semantics, thematic arguments are not treated as arguments to the verb, but are introduced by independent thematic role heads (Parsons 1990). Verbs are one-place predicates of events and are combined with the thematic arguments via a generalized predicate modification rule.

$$
\llbracket D O U_{\theta} \rrbracket=\lambda \operatorname{Cov} \lambda P_{\langle\varepsilon, t\rangle} \lambda e_{\varepsilon} \cdot \exists E_{\langle\varepsilon, t\rangle}\left[\forall e^{\prime} \in E \rightarrow \operatorname{Cov}\left(\theta\left(e^{\prime}\right)\right) \wedge P\left(e^{\prime}\right) \wedge e=\bigoplus E\right]
$$

$$
\begin{aligned}
& \text { Thematic cumulativity } \\
& \theta(\bigoplus E)=\bigoplus(\lambda x . \exists e \in E[\theta(e)=x])
\end{aligned}
$$

Let's apply the semantics of dou to the distributive use first. In example (3), repeated here as (31), we get a distributive reading when dou is indexed with agent thematic function. Suppose in the cover, each person by her/himself is an element. The resulting truth condition asserts that there is an event whose agent is they, and this event can be divided into a set of subevents, each of which is an event of lifting a piano with one person from they as its agent. 
A Unified Account of Three Uses of Dou

(31) Tamen dou taiqi le yi jia gangqin they DOU lift Asp one CL piano They each lifted a piano.



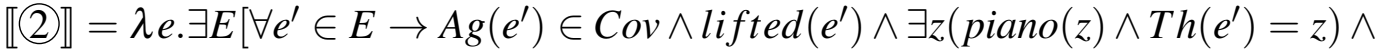
$e=\bigoplus E]$

【1)』 $=1$ iff $\exists e . A g(e)=\bigoplus$ they $\wedge \exists E\left[\forall e^{\prime} \in E \rightarrow A g\left(e^{\prime}\right) \in \operatorname{Cov} \wedge \operatorname{lifted}\left(e^{\prime}\right) \wedge \exists z(\operatorname{piano}(z) \wedge\right.$ $\left.\left.\operatorname{Th}\left(e^{\prime}\right)=z\right) \wedge e=\bigoplus E\right]$

The cumulative use of $d o u$ is captured straightforwardly too. In (7), repeated here as (32), the object precedes dou and becomes part of the description of the mereological sum event, not the subevents.

(32) San ge xuesheng ba wu ge pingguo dou chi le three CL students BA five CL apples DOU eat Asp Three students ate all of the five apples.

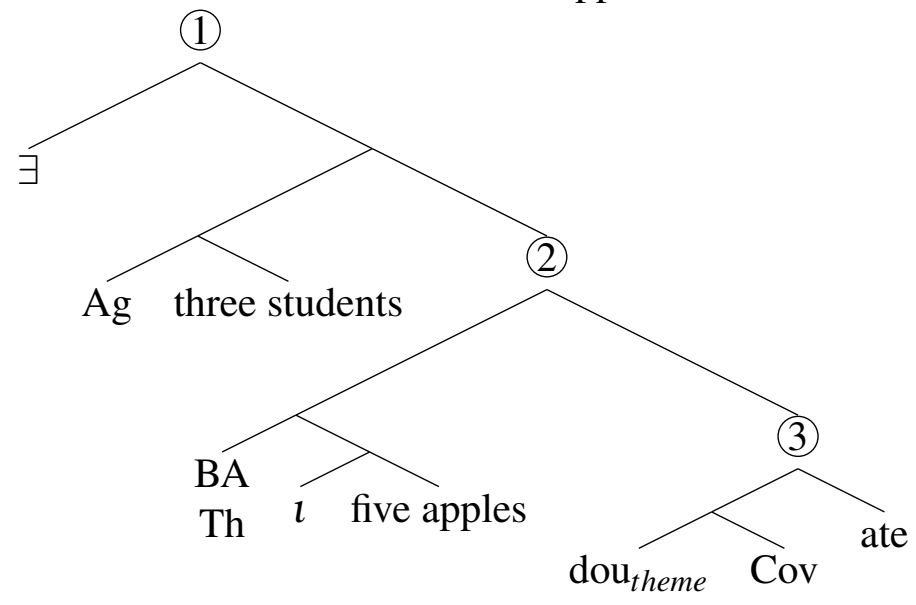

$$
\begin{aligned}
& \llbracket\left(3 \rrbracket=\lambda e . \exists E\left[\forall e^{\prime} \in E \rightarrow\left(T h\left(e^{\prime}\right) \in \operatorname{Cov} \wedge \text { ate }\left(e^{\prime}\right)\right) \wedge e=\bigoplus E\right]\right. \\
& \llbracket(2)=\lambda e . T h(e)=\bigoplus \text { the-five-apples } \wedge \exists E\left[\forall e^{\prime} \in E \rightarrow\left(T h\left(e^{\prime}\right) \in \operatorname{Cov} \wedge \text { ate }\left(e^{\prime}\right)\right) \wedge\right. \\
& e=\bigoplus E]
\end{aligned}
$$


【1)』 $=1$ iff $\exists e \exists X[$ students $(X) \wedge|X|=3 \wedge A g(e)=X \wedge T h(e)=\bigoplus$ the-five-apples $\wedge$ $\exists E\left[\forall e^{\prime} \in E \rightarrow\left(T h\left(e^{\prime}\right) \in \operatorname{Cov} \wedge\right.\right.$ ate $\left.\left.\left(e^{\prime}\right)\right) \wedge e=\bigoplus E\right]$

The resulting meaning we get is an unspecified meaning. It asserts that there is an event whose agent is three students and whose object is contextually salient five apples. This event can be divided into a set of subevents, each of which is an eating event whose theme is in the cover over the five apples. ${ }^{7}$ Since the agent of each subevent is not specified, how the five apples are divided between the students has multiple possibilities. Both the cumulative reading and the distributive reading will be true by this condition. Lumping these two readings, however, is not an undesirable result. As discussed in Kratzer 2007, distributive, collective and cumulative readings are not separate. She adopts the ellipsis test, replicated here in (33). This sentence is true when John and Mary individually lifted four boxes, but Bill and Sue cumulatively did so. ${ }^{8}$

(33) John and Mary lifted four boxes. Bill and Sue did too.

Last, the occasion use of dou is analyzed as a distributive reading over the temporal or location domain. In (1), repeated here as (34), dou is indexed with a temporal thematic function $\tau$ which takes the mereological sum event returns the temporal trace of the contextually salient horse races.

wo dou du le Laozhongshi
I DOU bet Asp Old Faithful
I bet on Old Faithful every time.

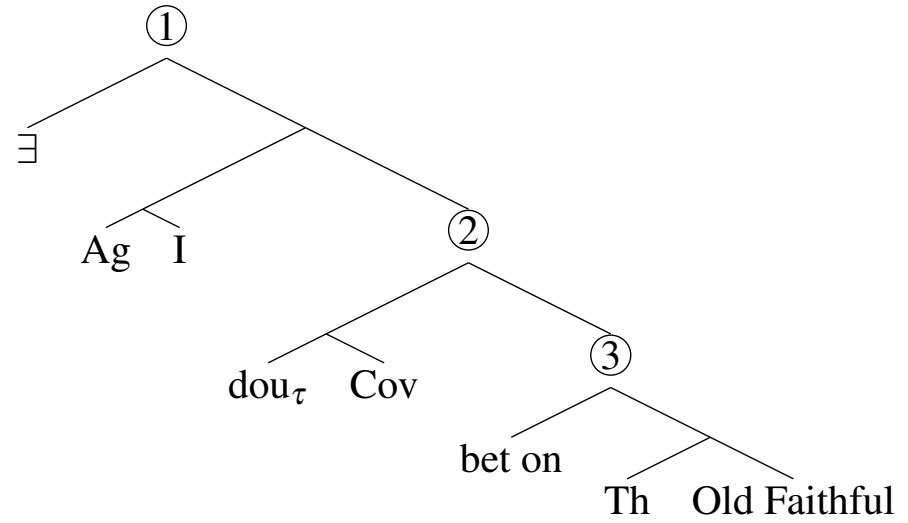

【(3) $\rrbracket=\lambda e \cdot T h(e)=O F \wedge$ bet-on $(e)$

$\llbracket(2) \rrbracket=\lambda e . \exists E\left[\forall e^{\prime} \in E \rightarrow\left(\tau\left(e^{\prime}\right) \in \operatorname{Cov} \wedge T h\left(e^{\prime}\right)=O F \wedge\right.\right.$ bet-on $\left.\left.\left(e^{\prime}\right)\right) \wedge e=\bigoplus E\right]$

7 Some readers may ask why dou is not indexed with the agent in the sentence. Recall from footnote 5 that an intervening BA-phrase confines the association of dou to the NP within it.

8 There is disagreement on the claim that distributive, collective, and cumulative readings are not separate. See discussion on this point in Champollion (forthcoming). 
【1]』 $=1$ iff $\exists e . A g(e)=I \wedge \exists E\left[\forall e^{\prime} \in E \rightarrow\left(\tau\left(e^{\prime}\right) \in \operatorname{Cov} \wedge T h\left(e^{\prime}\right)=O F \wedge\right.\right.$ bet-on $\left.\left(e^{\prime}\right)\right) \wedge$ $e=\bigoplus E]$

The reading we get asserts that there is an event whose agent is the speaker and this event can be divided into a set of subevents, each being a betting event with Old Faithful as its theme. Moreover, the run time of each subevent is in the contextually salient cover. Since the cover is on the temporal traces of the multiple horse races, the sum of the cover is the same as the sum of the run time of those horse races.

The composition above shows that analyzing dou as an event quantifier that always distributes through the mediation of events can unify the familiar use of distribution over NPs, the cumulative use and the occasion use. Next, we will see how this analysis can answer the questions we in $\S 1$.

\subsection{Answers to the questions posed in $\S 1$}

The semantics given to dou in (29) makes it flexible for dou to choose the plural element that it is associated with. Depending on the theta function that $d o u$ is indexed with, the associate can be a plural NP or a plurality of events. This flexibility answers our questions on sentences (1) to (3). Sentence (1) only has occasion reading because there is no semantically plural NP available to be associated with dou. (2) is only felicitous with occasion reading because the predicate meet requires a plurality of at least two people as its subject. Therefore, John and Mary predicated by meet is semantically singular. Dou needs to seek a plurality in the event domain and the occasion reading is obligatory.

In (4), dou can only associate with the subject plural NP when an individuallevel predicate is used. As observed in Kratzer 1995, temporal adverbs or location adverbs cannot modify individual-level predicates because they denote a set of states that do not have subparts varying with time or location, as shown in (35). Therefore, the occasion reading with individual-predicates is prohibited, see (5). This explains why (4) only has a distributive reading on the subject NP.

*Almost all swans are black in Australia.

In (6), the predicate break that cup denotes a one-time event. A singular subject NP forces an occasion reading, which, however, is not allowed by the predicate. Hence the ungrammaticality of this sentence. A plural subject is no help because a distributive reading on the subject requires the event denoted by the predicate to happen more than once, which again is not allowed in (6).

As for (7), we have seen in (32) that the semantics of dou derives an unspecified meaning for this sentence, which allows both a distributive reading on the object or a cumulative reading. Last, the contrast between (3) and (8) is a direct result of the 
dou's semantics. Since everything in the scope of dou becomes the description of the subevents, an object in its canonical position as in (8) will be part of the description of the subevents. Therefore, it is impossible to derive a cumulative reading between the subject NP and the object NP. Only the distributive reading over the subject NP is allowed.

\subsection{Two predictions}

Dou divides a sentence into two parts. Inside the scope of dou is the description of the subevents; outside the scope of dou is the description of the mereological sum event. In our cumulative example, if we insert an existential quantifier in the scope of $d o u$, we expect to see a distributive relationship between the theme and the existential quantifier. ${ }^{9}$ This is exactly what we find. In (36), suppose the contextually determined cover on the theme five apples divides this plurality into singular apples. Dou then asserts that each subevent takes one of the five apples as its theme. Moreover, in each subevent, the theme is consumed one bite. The correlation between the relative order of these constituents and the cumulative/distributive relationship between them follows naturally from dou's semantics.

san ge xuesheng ba wu ge pingguo dou chi le yikou three CL students BA five CL apples DOU eat Asp one bite Three students ate the five apples and each apple was consumed one bite.

$$
\begin{aligned}
& \llbracket(36) \rrbracket=\exists e \exists X[\text { students }(X) \wedge|X|=3 \wedge A g(e)=X \wedge T h(e)=\bigoplus \text { the-five- } \\
& \text { apples }] \wedge \exists E\left[\forall e^{\prime} \in E \rightarrow\left(T h\left(e^{\prime}\right) \in \operatorname{Cov} \wedge \text { ate-one-bite }\left(e^{\prime}\right)\right) \wedge e=\bigoplus E\right]
\end{aligned}
$$

A second prediction is the possibility of having more than one dou in a sentence. Suppose we have a plural NP outside of the scope of $d o u$ and the predicate is one that allows occasion reading. We should be able to insert two dous, one associated with the plural NP and one with the salient temporal/location traces. (39) show that this is possible.

Tamen dou shi dou du le Laozhongshi

they DOU SHI DOU bet on Asp Old Faithful

They each bet on Old Faithful each time/at each place.

\section{Discussion and conclusion}

I propose a treatment of dou following Champollion's (2016) cross-linguistic analysis of distance distributive items. Dou is analyzed as an event quantifier that always distributes through the mediation of events. It is shown that the distributive use,

9 I thank Lucas Champollion for his suggestion on this testing sentence. 
occasion use and cumulative use of $d o u$ are all captured by this proposal.

Taking stock of what we have seen so far, dou always seeks a plurality to be associated with, be it a plurality in the nominal domain or event domain. Although the current paper does not address the even use of $d o u$, the close connection between $d o u$ and plurality leads to a natural idea that focus is another way of introducing plurality. Actually, there exists an analysis along this line (Portner 2002) that analyzes dou as a universal quantifier over the alternative set. However, some linguists criticize this analysis based on the fact that dou can be used as even without any of the alternatives other than the prejacent being true (Chen 2008; Rullmann 1997: a.o). I will not provide a detailed discussion on the divergences, but would like to point out one problematic prediction of the criticism against Portner (2002). If it is true that none of the alternatives to the prejacent of dou is required to be true, sentence (39) should be grammatical, but it is not. Notice that the intended meaning is not infelicitous. It asserts that Zhangsan killed Lisi and presupposes that Zhangsan is the least likely person among the contextually salient people to do so.

Zhangsan dou shasi le Lisi

Zhangsan DOU kill Asp Lisi

Intended meaning: *Even Zhangsan killed Lisi.

Recent research on dou (Liao \& Jheng 2015) also questions the argument that the additive presupposition of $d o u$ in its even use is an illusion. In order to extend my analysis of dou to its even use, I will have to dismiss the arguments against the additive presupposition of $d o u$, but this will have to be left for future research.

\section{References}

Brisson, Christine. 1998. Distributivity, maximality and floating quantifiers. New Brunswick, NJ: Rutgers University PhD dissertation.

Champollion, Lucas. 2016. Overt distributivity in algebraic event semantics. Semantics and Pragmatics 9(16). 1-65. doi:10.3765/sp.9.16.

Champollion, Lucas. forthcoming. Distributivity, collectivity and cumulativity. In Hotze Rullmann Lisa Matthewson, Cécile Meier \& Thomas Ede Zimmermann (eds.), Blackwell Companion to Semantics, Wiley.

Chen, Liping. 2008. Dou: Distributivity and beyond. New Brunswick, NJ: Rutgers University $\mathrm{PhD}$ dissertation.

Cheng, Lisa Lai-Shen. 1994. Wh-words as polarity items. In Paul Jen-Kuei Li, Chu-Ren Huang \& C.-C. Jane Tang (eds.), Chinese Languages and Linguistics, vol. 2, 615-640. Taipei: Academia Sinica.

Cheng, Lisa Lai-Shen \& Anastasia Giannakidou. 2013. The non-uniformity of 
wh-indeterminates with polarity and free choice in Chinese. Strategies of Quantification 44. 123-151. doi:10.1093/acprof:oso/9780199692439.003.0007.

Dowty, David. 1987. Collective predicates, distributive predicates and all. In The Eastern States Conference on Linguistics (ESCOL) 3, 97-115.

Heim, Irene. 1982. The Semantics of Definite and Indefinite Noun Phrases. Amherst, MA: Umass $\mathrm{PhD}$ dissertation.

Higginbotham, James. 1985. On semantics. Linguistic Inquiry 16(4). 547-593.

Higginbotham, James. 1989. Elucidations of meaning. Linguistics and Philosophy 12(4). 465-517. doi:10.1007/bf00632473.

Huang, Cheng-Teh James, Yen-hui Audrey Li \& Yafei Li. 2009. The ba construction. In Cheng-Teh James Huang, Yen-hui Audrey Li \& Yafei Li (eds.), The Syntax of Chinese, vol. 8, Cambridge University Press Cambridge. doi:10.1017/cbo9781139166935.006.

Huang, Shi-Zhe. 1996. Predication and Qantification in Mandarin Chinese: A Case Study of Dou. Philadelphia, PA: University of Pennsylvania PhD dissertation.

Kratzer, Angelika. 1995. Stage-level and individual-level predicates. In Gregory N. Carlson \& Francis Jeffry Pelletier (eds.), The Generic Book, 125-175. Chicago/London: The University of Chicago Press.

Kratzer, Angelika. 2002. The event argument and the semantics of verbs, chapter 2. Manuscript. Amherst: University of Massachusetts, Amherst, MA.

Kratzer, Angelika. 2007. On the plurality of verbs. In Martin Schäfer Johannes Dölling, Tatjana Heyde-Zybatow (ed.), Event Structures in Linguistic Form and Interpretation, 269-300. Berlin, Germany: Mouton de Gruyter. doi:10.1515/9783110925449.269.

Landman, Fred. 2000. Events and Plurality: The Jerusalem Lectures, vol. 76. Springer Science \& Business Media. doi:10.1007/978-94-011-4359-2.

Lee, Thomas Hun-tak. 1986. Studies on Quantification in Chinese. Los Angeles, CA: UCLA PhD dissertation.

Li, Xiao-guang. 1995. Dou as event quantifier. In The North American Conference on Chinese Linguistics (NACCL) 7 and the International Conference on Chinese Linguistics (ICCL) 4, 1-19. Madison, WI: University of Wisconsin-Madison.

Liao, H. C. 2011. Alternatives and Exhaustification: Non-interrogative Uses of Chinese Wh-words. Cambridge, MA: Harvard University PhD dissertation.

Liao, Wei-wen Roger \& Wei-cheng Sam Jheng. 2015. How do we get even. In The International Workshop on Theoretical East Asian Linguistics (TEAL) 10, 13-14. Tokyo University of Foreign Studies.

Lin, Jo-Wang. 1998. On existential polarity wh-phrases in Chinese. Journal of East Asian Linguistics 7(3). 219-255.

Link, Godehard. 2002. The logical analysis of plurals and mass terms: A lattice-theoretical approach. In Portner PH \& Partee BH (ed.), For- 
A Unified Account of Three Uses of Dou

mal Semantics: The Essential Readings, 127-146. John Wiley \& Sons. doi:10.1002/9780470758335.ch4.

Liu, Mingming. 2017. Varieties of alternatives: Mandarin focus particles. Linguistics and Philosophy 40(1). 61-95. doi:10.1007/978-981-10-6208-7_3.

Parsons, Terence. 1990. Events in the Semantics of English, vol. 5. Cambridge, Ma: MIT Press.

Portner, Paul. 2002. Topicality and (non-) specificity in Mandarin. Journal of Semantics 19(3). 275-287. doi:10.1093/jos/19.3.275.

Rullmann, Hotze. 1997. Even, polarity, and scope. In Wiebe G. \& Libben G. Gibson M. (ed.), Papers in Experimental and Theoretical Linguistics, vol. 4 40-64, Edmonton: University of Alberta.

Schwarzschild, Roger. 1996. Pluralities, vol. 61. Springer Science \& Business Media. doi:10.1007/978-94-017-2704-4.

Xiang, Ming. 2008. Plurality, maximality and scalar inferences: A case study of Mandarin dou. Journal of East Asian Linguistics 17(3). 227-245. doi:10.1007/s10831-008-9025-9.

Zhuo Chen

Linguistics Program

The Graduate Center, CUNY

365 Fifth Avenue

New York, NY 10016

zchen3@gradcenter.cuny.edu 